OPEN ACCESS

Edited by:

Yasuko Tsunetsugu Yokota, Tokyo University of Technology, Japan

Reviewed by: Tetsuo Nakayama, Kitasato University, Japan Masaya Sugiyama, National Center for Global Health and Medicine, Japan

${ }^{*}$ Correspondence: Yoshio Mori yoshiom@nih.go.jp

Specialty section: This article was submitted to Virology,

a section of the journal Frontiers in Microbiology

Received: 20 June 2017 Accepted: 27 July 2017 Published: 09 August 2017

Citation:

Mori Y, Miyoshi M, Kikuchi M, Sekine M, Umezawa M, Saikusa M, Matsushima $Y$, Itamochi M, Yasui $Y$, Kanbayashi D, Miyoshi T, Akiyoshi K

Tatsumi C, Zaitsu S, Kadoguchi M, Otsuki N, Okamoto K, Sakata M, Komase K and Takeda M (2017) Molecular Epidemiology of Rubella Virus Strains Detected Around the Time of the 2012-2013 Epidemic

in Japan. Front. Microbiol. 8:1513. doi: 10.3389/fmicb.2017.01513

\section{Molecular Epidemiology of Rubella Virus Strains Detected Around the Time of the 2012-2013 Epidemic in Japan}

Yoshio Mori1*, Masahiro Miyoshi², Masayuki Kikuchi', Masao Sekine ${ }^{4}$, Masahiro Umezawa ${ }^{5}$, Miwako Saikusa ${ }^{6}$, Yuki Matsushima7 ${ }^{7}$, Masae Itamochi ${ }^{8}$, Yoshihiro Yasui ${ }^{9}$, Daiki Kanbayashi ${ }^{10}$, Tatsuya Miyoshi ${ }^{11}$, Kyoko Akiyoshi12, Chika Tatsumi ${ }^{13}$, Shuichi Zaitsu ${ }^{14}$, Mayumi Kadoguchi ${ }^{15,16}$, Noriyuki Otsuki', Kiyoko Okamoto ${ }^{1}$, Masafumi Sakata ${ }^{1}$, Katsuhiro Komase ${ }^{1,17}$ and Makoto Takeda ${ }^{1}$

${ }^{1}$ Department of Virology 3, National Institute of Infectious Diseases, Tokyo, Japan, ${ }^{2}$ Hokkaido Institute of Public Health, Sapporo, Japan, ${ }^{3}$ Sapporo City Institute of Public Health, Sapporo, Japan, ${ }^{4}$ Sendai City Institute of Public Health, Sendai, Japan, ${ }^{5}$ Ibaraki Prefectural Institute of Public Health, Ibaraki, Japan, ${ }^{6}$ Yokohama City Institute of Public Health, Yokohama, Japan, ${ }^{7}$ Kawasaki City Institute for Public Health, Kawasaki, Japan, ${ }^{8}$ Toyama Institute of Health, Toyama, Japan, ${ }^{9}$ Aichi Prefectural Institute of Public Health, Nagoya, Japan, ${ }^{10}$ Osaka Institute of Public Health, Osaka, Japan, ${ }^{11}$ Sakai City Institute of Public Health, Sakai, Japan, ${ }^{12}$ Kobe Institute of Health, Kobe, Japan, ${ }^{13}$ Shimane Prefectural Institute of Public Health and Environmental Science, Shimane, Japan, ${ }^{14}$ Fukuoka City Institute of Health and Environment, Fukuoka, Japan, ${ }^{15}$ Kumamoto City Environmental Research Center, Kumamoto, Japan, ${ }^{16}$ Kumamoto City Hospital, Kumamoto, Japan, ${ }^{17}$ Infectious Disease Surveillance Center, National Institute of Infectious Diseases, Tokyo, Japan

A nationwide rubella epidemic occurred from 2012 to 2013 in Japan, resulting in around 17,000 rubella cases and the birth of 45 infants with congenital rubella syndrome. The aim of this study was to genetically characterize the rubella viruses (RVs) circulating around the time of the epidemic in Japan. In total, $221 \mathrm{RV}$ strains detected from 14 prefectures in Japan between 2010 and 2014 were sequenced in the 739 nucleotide-window region within the E1 gene. The virus strains were chronologically and geographically characterized into groups based on phylogenetic analysis. Among the 221 strains analyzed, 192 (87\%), 26 (12\%), and 3 (1\%) strains were classified into genotypes $2 \mathrm{~B}, 1 \mathrm{E}$, and $1 \mathrm{~J}$, respectively. The majority $(n=184)$ of the genotype $2 \mathrm{~B}$ strains belonged to lineage $2 \mathrm{~B}-\mathrm{L} 1$ and shared nucleotide homology with the strains detected in Southeast and East Asian countries. Phylogenetic analyses demonstrated that at least six distinct clusters of RV strains (clusters 1-6) induced outbreaks in Japan between 2010 and 2014. Among them, strains from clusters 3, 4, and 6 circulated almost simultaneously during 2012-2013. The cluster 3 strains circulated locally, whereas strains from cluster 4 spread nationwide. The findings suggest that RVs were introduced into Japan many times from neighboring countries. The 2012-2013 epidemic was a complex of outbreaks induced by at least three clusters of RV strains.

Keywords: rubella virus, molecular epidemiology, genotype, epidemic, Japan

Abbreviations: CRS, congenital rubella syndrome; $M R$ vaccine, measles and rubella combination vaccine; RV, rubella virus; WHO, World Health Organization. 


\section{INTRODUCTION}

Rubella is caused by infection with rubella virus (RV) and usually presents as a mild illness characterized by low-grade fever, a short-lived morbilliform rash, and lymphadenopathy (Reef and Plotkin, 2012). The most serious concern with this disease is that the infection to pregnant women early during their pregnancy may result in miscarriage, stillbirth, or infants born with birth defects known as congenital rubella syndrome (CRS).

The live-attenuated rubella vaccines available at present are highly effective in preventing and controlling CRS as well as rubella (Reef and Plotkin, 2012). In Japan, a single-dose rubella vaccination was introduced into the national immunization program, targeting girls in junior high schools in 1977 (Saitoh and Okabe, 2014). From 1989, children aged 12-72 months were able to receive measles vaccination using a domestic measles, mumps, and rubella combination vaccine, however, the vaccine was withdrawn in 1993 due to the relatively high incidence of meningitis caused by the mumps component (Ueda et al., 1995). In 1995, the targets of the rubella vaccination were changed to include boys and girls aged 12-90 months. Additionally, boys and girls in junior high schools were also included to the targets as a temporary measure. However, the vaccination coverage at this generation was lower than $60 \%$ (Ministry of Health, Labor, and Welfare, Japan, 2017). In 2006, a two-dose vaccination of 1 - to 2 - and 5- to 7 -year-old children using a measles and rubella combination vaccine (MR vaccine) was introduced. Furthermore, to ensure immunization against both diseases among adolescents, a catch-up MR vaccination was implemented targeting two cohorts, those aged 12-13 and 17-18 years between 2008 and 2013. The assessment of population immunity against rubella as part of the National Epidemiological Surveillance of Vaccine-Preventable Diseases program showed that children and adolescents aged 2-24 years old and adult females had $\geq 90 \%$ population immunity against rubella as of 2012 (Tuberculosis and Infectious Diseases Control Division, Ministry of Health, Labor, and Welfare, Japan, and Infectious Disease Surveillance Center, National Institute of Infectious Diseases, 2015). However, up to $25 \%$ of adult males remained susceptible to rubella, in particular those in their $30 \mathrm{~s}$ to $50 \mathrm{~s}$, because they had not received rubella vaccination by the routine immunization program. Since case-based surveillance of rubella started in 2008 in Japan, a low number of rubella cases were reported before 2011. However, the nationwide epidemic that occurred in 2012-2013, resulting in reports of approximately 17,000 rubella cases (Tanaka-Taya et al., 2013; Saitoh and Okabe, 2014; Ujiie et al., 2014; National Institute of Infectious Diseases, and Tuberculosis, and Infectious Diseases Control Division, Ministry of Health, Labor, and Welfare, Japan, 2015, 2016). Associated with this epidemic, a total of 45 CRS cases were reported in 2012-2014. This epidemic mainly affected males in their 30 s to 50 s, who had not received rubella vaccination by the routine immunization program, and males and females in their 20 s, whose vaccination coverage was relatively low despite getting an opportunity to receive one or two dose rubella vaccination. The total of these demographic groups accounted for about $80 \%$ of the rubella patients in 2013.
RV belongs to the genus Rubivirus in the family Togaviridae (Hobman, 2013). The virion is enveloped by a lipid membrane and possesses a positive-sense single-stranded RNA genome of approximately $9.8-\mathrm{kb}$. The genome contains two openreading frames that encode the non-structural proteins, p150 and p90, and the structural proteins, C, E2, and E1. The envelope glycoprotein $\mathrm{E} 1$ is involved in receptor binding (Cong et al., 2011) and membrane fusion (DuBois et al., 2013; Dube et al., 2014) and is the predominant antigen eliciting neutralizing or hemagglutination-inhibiting antibodies (Wolinsky et al., 1991).

The Global Vaccine Action Plan 2011-2020 endorsed by the World Health Assembly in 2012 indicated that measles and rubella were targeted for elimination in at least five World Health Organization (WHO) regions by 2020 (The Decade of Vaccine Collaboration, 2012). The surveillance of $\mathrm{RV}$ using molecular analysis is recognized to be important for characterizing circulating viruses in endemic countries, confirming the disappearance of endemic strains at the eliminating or eliminated stage, and tracing the transmission of newly imported strains. The Global Measles and Rubella Laboratory Network encourages genetic classification based on analysis of a 739-nucleotide window region within the $E 1$ gene for virological surveillance (Mulders et al., 2016). RV strains are classified into two large clades, 1 and 2, that are further divided into $10(1 \mathrm{a}, 1 \mathrm{~B}, 1 \mathrm{C}, 1 \mathrm{D}, 1 \mathrm{E}, 1 \mathrm{~F}, 1 \mathrm{G}, 1 \mathrm{H}, 1 \mathrm{I}$, and $1 \mathrm{~J})$ and three genotypes (2A, 2B, and 2C), respectively (WHO, 2013b). At present, most of the currently circulating wild-type RV strains belong to one of only four genotypes (1E, 1G, 1J, and 2B), with genotypes $1 \mathrm{E}$ and $2 \mathrm{~B}$ RV strains being frequently detected worldwide (Abernathy et al., 2011; WHO, 2013b; Mulders et al., 2016; Rivailler et al., 2017). Sub-division of these genotypes has been proposed to improve the resolution of genetic classification (Rivailler et al., 2017).

In this study, we genetically characterized the $221 \mathrm{RV}$ strains detected around the time of the 2012-2013 epidemic in Japan to provide insight into the epidemiology of these strains.

\section{MATERIALS AND METHODS}

\section{Nucleotide Sequencing of the 739-Nucleotide Window Region within the E1 Gene}

Between 2010 and 2014, clinical samples (including throat swab, blood, and urine) from suspected rubella and CRS patients were collected from patients in 14 prefectures (Hokkaido, Miyagi, Ibaraki, Tokyo, Chiba, Kanagawa, Toyama, Aichi, Osaka, Hyogo, Kagawa, Shimane, Fukuoka, and Kumamoto) and sent to local or national laboratories to detect $\mathrm{RV}$ as part of the national infectious agents surveillance program. Criteria for the sample collection from suspected cases with rubella or CRS varied by local government but all the samples sent to the laboratories were analyzed in principle. Samples positive for RV were subjected to nucleotide sequencing of the WHOrecommended 739-nucleotide window region within the E1 
gene (nucleotides 8731-9469) as follows. Briefly, the cDNA was synthesized using a commercial reverse transcription kit and random hexamers as primer and extracted viral RNA as template. The nucleotide region containing the 739-nucleotide window was amplified as two overlapping fragments by nestedPCR (fragment 1: nucleotides 8664-9129, fragment 2: nucleotides 9070-9492). For amplification of fragment 1, the first PCR primer set (E1-2F: 5'-AGC GAC GCG GCC TGC TGG GG-3' and E1-2R: 5'-CCA GCG CGT ATG TGG AGT CC- $3^{\prime}$ ) and the nested PCR primer set (E1-6F: $5^{\prime}$-ACA CCG TGA TGA GCG TGT TC-3' and E1-10R: $5^{\prime}$-ATG TGG AGT CCG CAC TTG CG- $\left.3^{\prime}\right)$ were used. For amplification of fragment 2, the first PCR primer set (E1-7F: 5'-AGC GAC GCG GCC TGC TGG GG-3' and E1-12R: 5'-TGT GTG CCA TAC ACC ACG CC-3') and the nested PCR primer set (E1-3F: 5'-CGG CGA GGT GTG GGT CAC GC-3' and E1-3R: 5'-ACC CGC GCG CTC GCG CGA TC- $3^{\prime}$ ) were used. After purification of these fragments, the nucleotide sequences were determined by a fluorescent dyeterminator cycle sequencing method using the primers E1-6F or E1-10R and E1-3F or E1-3R for fragments 1 and 2, respectively. The nucleotide sequences of the two fragments were assembled to obtain the whole sequence of the 739-nucleotide window region. The nucleotide sequences of RV strains determined in this study were submitted to the GenBank database under the accession numbers indicated in Supplemental Table 1.

\section{Phylogenic Analysis}

Phylogenetic analysis of the sequence data obtained in this study, together with those of the genotype reference strains (WHO, 2013b), the proposed lineage reference strain candidates (Rivailler et al., 2017), and representative strains detected in other countries (Supplemental Tables 2, 3), was conducted using the MEGA program version 6.0.6. Phylogenetic trees were constructed by the maximum-likelihood method using the Tamura-Nei model (Tamura and Nei, 1993). The reliability of the tree at each branch node was assessed by the bootstrap method with 1,000 replicates. The genotype of RV strains was determined based on the phylogenetic tree topology constructed with the genotype reference strains (WHO, 2013b).

TABLE 1 | Number of rubella viruses (RVs) analyzed in this study.

\begin{tabular}{lccccc}
\hline & & \multicolumn{3}{c}{ Number of RVs analyzed in this study } \\
\cline { 3 - 5 } & & \multicolumn{2}{c}{ Genotype } & \\
\cline { 3 - 5 } Year & Number of rubella cases & $\mathbf{1 E}$ & $\mathbf{1 J}$ & $\mathbf{2 B}$ & Total \\
\hline 2010 & 87 & 1 & 1 & 3 & 5 \\
2011 & 378 & 7 & 2 & 23 & 32 \\
2012 & 2,386 & 11 & 0 & 63 & 74 \\
2013 & 14,344 & 7 & 0 & 95 & 102 \\
2014 & 319 & 0 & 0 & 8 & 8 \\
Total & 17,514 & 26 & 3 & 192 & 221 \\
\hline
\end{tabular}

${ }^{1}$ Reference: National Institute of Infectious Diseases, and Tuberculosis, and Infectious Diseases Control Division, Ministry of Health, Labor, and Welfare, Japan (2016).

\section{Ethics Statement}

According to the Law Concerning the Prevention of Infectious Diseases and Medical Care for Patients of Infections in Japan, rubella and CRS are defined as notifiable infectious diseases, and specimens from patients suspected of having rubella or CRS could be collected and tested for RVs without informed consent from the patients. The Ethics Committee of the National Institute of Infectious Diseases agreed to the publishing of this paper (No. 761).

\section{RESULTS}

\section{Genotyping of RV Strains Detected in Japan between 2010 and 2014}

To characterize the RV strains circulating around the time of the 2012-2013 epidemic, the nucleotide sequences of the 739nucleotide window region of 221 strains detected from rubella $(n=216)$ and CRS patients $(n=5)$ in 14 prefectures between 2010 and 2014 were determined and analyzed (Tables 1, 2). The number of analyzed RV strains in each prefecture was not necessarily proportionate to the number of rubella and CRS cases. The clinical background was available for $97.7 \%$ of the rubella patients $(n=211)$. The distribution of them by gender and age are indicated in Table 3. The ratio of male was $75.6 \%$, and that of adults ( $\geq 20$-year-old) was $83.7 \%$. These distributions were quite similar to those of the endemic mass population in 2013 (TanakaTaya et al., 2013; National Institute of Infectious Diseases, and Tuberculosis, and Infectious Diseases Control Division, Ministry of Health, Labor, and Welfare, Japan, 2015, 2016).

TABLE 2 | Number of analyzed rubella viruses (RVs) by geographic area of detection and by year.

\begin{tabular}{|c|c|c|c|c|c|c|}
\hline \multirow{3}{*}{$\frac{\text { Geographic area }}{\text { Prefecture }}$} & \multicolumn{6}{|c|}{ Number of RVs } \\
\hline & \multicolumn{5}{|c|}{ Year } & \multirow[b]{2}{*}{ Total } \\
\hline & 2010 & 2011 & 2012 & 2013 & 2014 & \\
\hline Hokkaido a & 0 & 2 & 1 & 9 & 1 & 13 \\
\hline Miyagib & 0 & 0 & 0 & 1 & 0 & 1 \\
\hline Ibarakic $^{\mathrm{c}}$ & 0 & 1 & 0 & 0 & 0 & 1 \\
\hline Tokyo ${ }^{d}$ & 0 & 1 & 0 & 0 & 0 & 1 \\
\hline Chiba $^{d}$ & 0 & 0 & 1 & 0 & 0 & 1 \\
\hline Kanagawa ${ }^{b}$ & 4 & 12 & 29 & 35 & 5 & 85 \\
\hline Toyama $^{c}$ & 1 & 0 & 0 & 0 & 0 & 1 \\
\hline Aichic $^{\mathrm{C}}$ & 0 & 1 & 12 & 20 & 2 & 35 \\
\hline Osaka ${ }^{a}$ & 0 & 6 & 4 & 6 & 0 & 16 \\
\hline Hyogo ${ }^{b}$ & 0 & 0 & 19 & 22 & 0 & 41 \\
\hline Kagawa $^{d}$ & 0 & 0 & 1 & 0 & 0 & 1 \\
\hline Shimane ${ }^{c}$ & 0 & 0 & 0 & 7 & 0 & 7 \\
\hline Fukuoka $^{b}$ & 0 & 8 & 7 & 2 & 0 & 17 \\
\hline Kumamoto $^{\text {b }}$ & 0 & 1 & 0 & 0 & 0 & 1 \\
\hline
\end{tabular}

a Data from the public health laboratories in both the prefecture and the city designated by government ordinance. ${ }^{b}$ Data from the public health laboratory(ies) in the city(ies) designated by government ordinance. ${ }^{c}$ Data from the public health laboratory in the prefecture. ${ }^{\mathrm{d}}$ Data from the National Institute of Infectious Diseases. 
Genotyping analysis indicated that these virus strains were classified into one of three genotypes, $1 \mathrm{E}, 1 \mathrm{~J}$, and $2 \mathrm{~B}$. The majority ( $n=192 ; 87 \%$ ) of these virus strains were of genotype $2 \mathrm{~B}$, with only $12 \%(n=26)$ belonging to genotype $1 \mathrm{E}$. The genotype $1 \mathrm{~J}$ strains $(n=3)$ were only detected until 2011.

\section{Phylogenetic Tree Analysis of the Genotype 2B Strains}

Phylogenetic tree analysis was conducted using a dataset comprising the nucleotide sequences of the genotype $2 \mathrm{~B}$ strains detected in Japan between 2010 and $2014(n=192)$ and representative $2 \mathrm{~B}$ strains detected from 20 countries or regions ( $n=56$, Supplemental Table 2) (Figure 1). Almost all of the strains detected in Japan were classified into two lineages, 2B-L1 and 2B-L2c. Of the genotype 2B strains, 96\% $(n=184)$ belonged to lineage $2 \mathrm{~B}-\mathrm{L} 1$, which was comprised strains detected in seven countries or regions (mainland China, Hong Kong, Vietnam, Malaysia, Thailand, Iran, and United Kingdom) mainly in Southeast and East Asia, suggesting that the majority of RV strains detected in Japan originated from these areas (Figure 1A). Lineage 2B-L2C comprised strains that originated in Europe, Africa, and Asia (Figure 1A).

In the phylogenetic tree, the RV strains detected in Japan predominantly formed five distinct clusters (clusters 1-5), which were supported by over $40 \%$ of bootstrap values (Figures 1A-D). The RV strains of clusters 1 and 2 were mainly detected in 2010 and 2011, whereas those of clusters 3, 4, and 5 were detected from 2012 to 2014 . The initial strain within cluster 4 (RVs/Yokohama.JPN/17.12/) was detected from an imported case from Thailand (Figure 1D). Two strains detected from imported rubella cases from Japan (RVs/Ontario.CAN/14.13/ and RVs/Hawaii.USA/17.13/) belonged to cluster 4, which was consistent with the epidemiological information (Figure 1D).

\section{Phylogenetic Tree Analysis of the Genotype 1E Strains}

Phylogenetic analysis using a dataset consisting of nucleotide sequences from the genotype $1 \mathrm{E}$ strains detected in Japan between

TABLE 3 | Distribution by age and gender of rubella patients who had possessed the rubella virus strains analyzed in this study.

\begin{tabular}{lccc}
\hline Age group in years & Female & Male & Total (\%) \\
\hline$<1$ & 0 & 1 & $1(0.5)$ \\
$1-10$ & 5 & 6 & $11(5.3)$ \\
$11-19$ & 10 & 12 & $22(10.5)$ \\
Subtotal $(<19)$ & 15 & 19 & $34(16.3)$ \\
$20-29$ & 19 & 33 & $52(24.9)$ \\
$30-39$ & 7 & 64 & $71(34.0)$ \\
$40-49$ & 5 & 32 & $37(17.7)$ \\
$\geq 50$ & 5 & 10 & $15(7.2)$ \\
Subtotal $(\geq 20)$ & 36 & 139 & $175(83.7)$ \\
\hline Total (\%) & $51(24.4)$ & $158(75.6)$ & $209(100)$
\end{tabular}

Data from the rubella patients unavailable for their clinical background $(n=7)$ and the CRS patients $(n=5)$ were excluded.
2010 and $2013(n=26)$ and representative $1 \mathrm{E}$ strains detected in other countries or regions $(n=35$, Supplemental Table 3) showed that the strains detected in 2010-2011 and those in 2012-2013 were classified into different lineages (Figure 2). The genotype $1 \mathrm{E}$ strains detected in Japan between 2010 and 2011 did not form distinct clusters but were included in lineage 1E-L1, which comprised strains detected in, or imported from, China, Taiwan, Hong Kong, and Russia. The genotype 1E strains detected in Japan between 2012 and 2013 belonged to lineage 1E-L2, which comprised strains detected in, or imported from, Malaysia, Hong Kong, Indonesia, and Kazakhstan, and almost all of these strains formed "cluster 6," which was supported by 69\% of the bootstrap values.

\section{Time Course of Detection of the Strains within Different Genetic Clusters}

Phylogenetic analyses demonstrated that RV strains within at least six distinct genetic clusters (clusters 1-6) induced outbreaks in Japan between 2010 and 2014. Figure 3 shows the time course of detection of these RV strains. The RV strains in clusters 1 and 2 were detected between week 48 of 2010 and week 1 of 2012. By contrast, the RV strains in the remaining four clusters were detected overlappingly between 2012 and 2013. The strains in cluster 4, the largest cluster, were continuously detected throughout a 2-year period (from week 17 of 2012 to week 16 of 2014), whereas the cluster 3 and 6 strains were detected for approximately 1 year until the middle of 2013 (from week 13 of 2012 to week 22 of 2013 and from week 8 of 2012 to week 34 of 2013, respectively). These data indicated that the 2012-2013 epidemic was a complex of outbreaks induced by several RV strains of different origins.

\section{Geographic Distribution of the Strains of Different Genetic Clusters}

Figure 4 shows the detection rates of the RV strains among different genetic clusters by prefecture between 2010 and 2014 . The cluster 4 strains were detected in all seven prefectures where multiple RV strains were reported, suggesting that the RV strains were distributed throughout Japan. By contrast, the cluster 3 strains, the second largest group, were predominantly detected in Hyogo and Aichi prefectures and detection was limited in other prefectures, suggesting that this type of RV strain did not spread nationwide.

\section{DISCUSSION}

In Japan, a single dose rubella vaccination was first introduced to the routine vaccination program targeting to girls in junior high schools in 1977, however, this program could not sufficiently control endemic circulation of rubella with large epidemics occurring in cycles of about 5 years (Katow, 2004b). To overcome this situation, a single-dose rubella vaccination program and subsequently a two-dose MR vaccination program were introduced targeting both male and female children and adolescents. Following the introduction of these programs, the number of rubella and CRS cases dramatically decreased in 

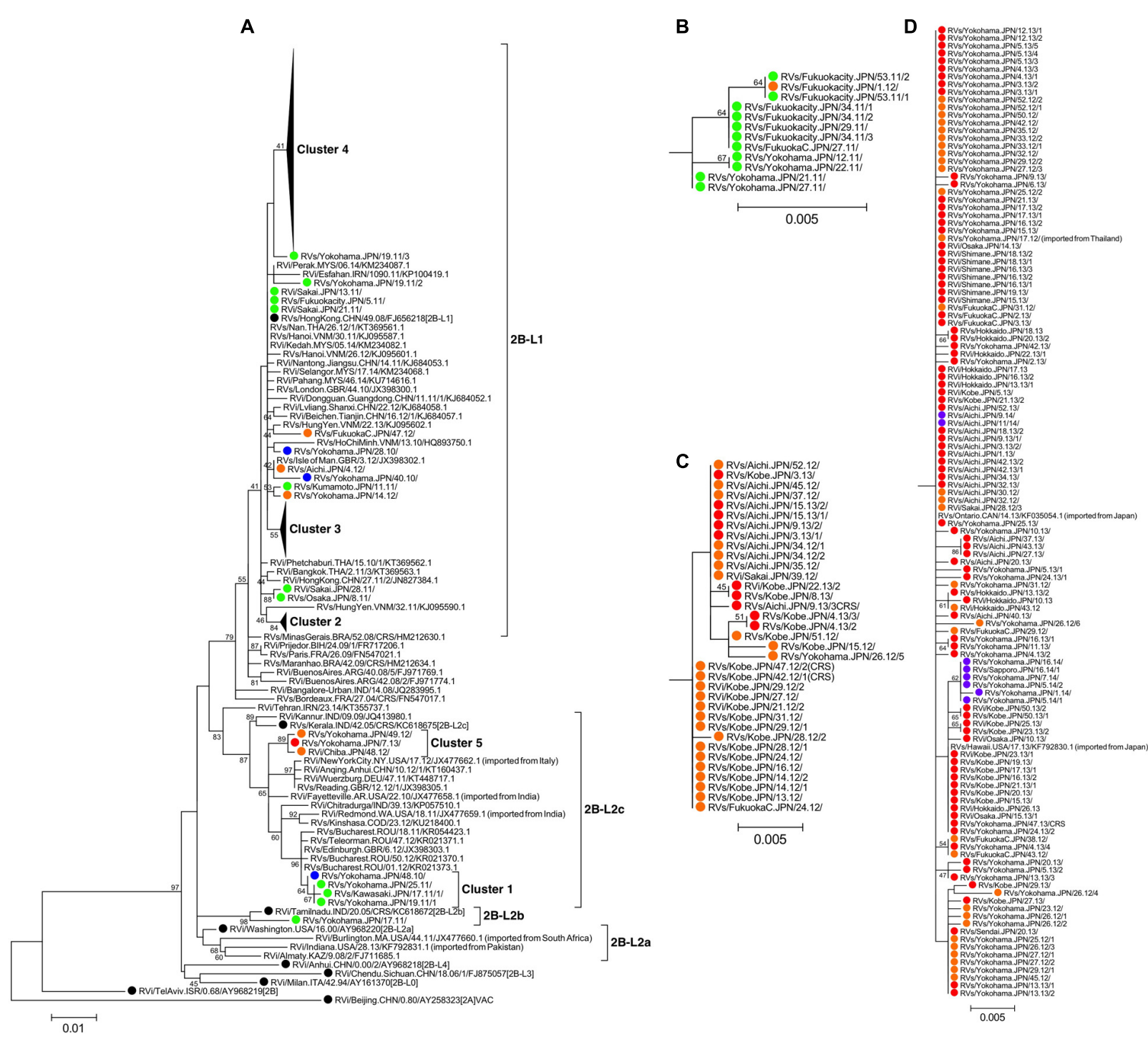

FIGURE 1 | Phylogenetic tree of the genotype 2B RV strains. The phylogenetic tree was constructed using the maximum likelihood method and was based on the sequence of the 739-nucleotide-window region within the E1 gene. Bootstrap values greater than $40 \%$ are shown adjacent to the corresponding nodes. (A) The entire phylogenetic tree for the genotype 2B strains. Filled triangles show clusters accumulating nodes of strains detected in Japan or exported from Japan. Panels (B-D) indicate the clusters 2, 3, and 4, respectively. Circles colored in blue, green, orange, red, and purple indicate strains detected in Japan in 2010, 2011, 2012, 2013, and 2014, respectively. The genotype-reference (16) and proposed lineage-reference strains (18) are indicated by black circles. The genotype $2 \mathrm{~A}$ reference strain (RVi/Beijing.CHN/0.80/[2A]VAC) is included as an out-group.

Japan, with the lowest annual number of rubella cases $(n=87)$ being achieved in 2010. Because there has been limited genotype information on RV strains in Japan prior to 2010, it was unclear which endemic strains had previously been circulating (Katow, 2004a). The original strains used to produce the Japanese domestic vaccine, which belonged to genotype $1 \mathrm{a}$, were isolated in the 1960s, and genotype 1D and 1J strains were detected in the 1990s and 2000s, respectively (Katow, 2004a; Otsuki et al., 2011; WHO, 2013b). In this study, no RV strains of genotype 1a wildtype or 1D were detected, and only three strains of genotype $1 \mathrm{~J}$ were detected up until 2011, suggesting that transmission of these genotype strains had already been interrupted in Japan.
Most of the RV strains detected in Japan between 2010 and 2014 were classified into either genotype $2 \mathrm{~B}$ or $1 \mathrm{E}$. This is similar to the reported global situation (Abernathy et al., 2011; Tran et al., 2012; Cheng et al., 2013; WHO, 2013b; Zhu et al., 2015; Mulders et al., 2016; Rivailler et al., 2017). The global convergence of currently circulating RV genotypes makes it difficult to distinguish imported strains from endemic strains and to understand the control status of $\mathrm{RV}$ in each country according to genotyping data alone. To overcome this, several studies have conducted sub-division of genotypes $2 \mathrm{~B}$ or $1 \mathrm{E}$ based on detailed phylogenetic analyses, although this modified classification system has not yet been 


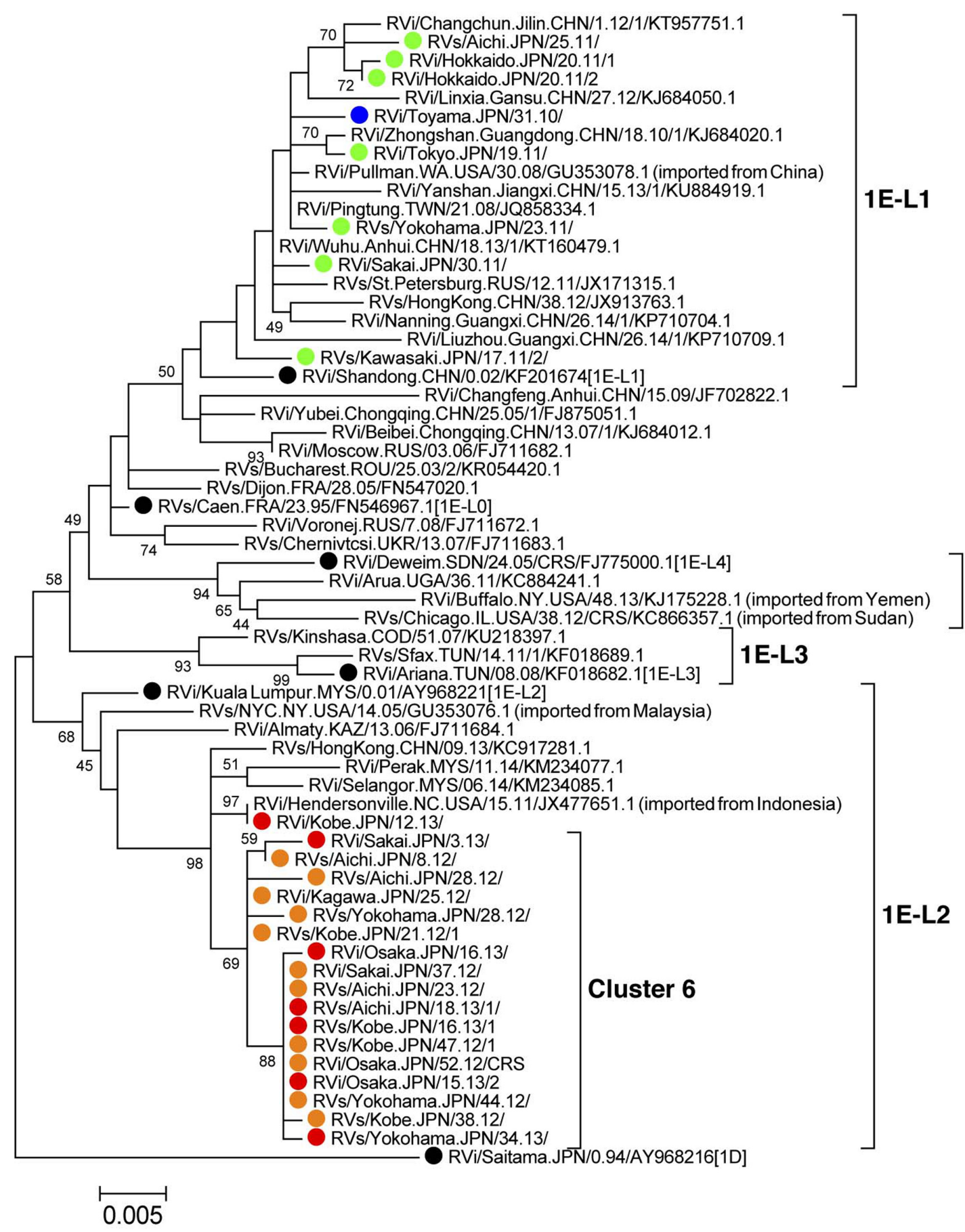

FIGURE 2 | Phylogenetic tree of the genotype 1E RV strains. The phylogenetic tree was constructed using the maximum likelihood method and was based on the sequence of the 739-nucleotide-window region within the E1 gene. Bootstrap values greater than $40 \%$ are shown adjacent to the corresponding nodes. Circles colored in blue, green, orange, and red indicate the strains detected in Japan in 2010, 2011, 2012, and 2013, respectively. The genotype-reference (16) and proposed lineage-reference strains (18) are indicated by black circles. The genotype 1D reference strain (RVi/Saitama.JPN/0.94/[1D]) is included as an out-group.

standardized (Zhu et al., 2012, 2015; Cheng et al., 2013; Rivailler et al., 2017). Rivailler et al. (2017) analyzed the largest sample of worldwide RV strains investigated to date, both genetically and geographically, and proposed precise sub-grouping of genotypes $1 \mathrm{E}, 1 \mathrm{G}$, and $2 \mathrm{~B}$, indicating reference strain candidates for each sub-group. In the present study, we analyzed and classified RV strains detected in Japan between 2010 and 2014 according to this sub-grouping. 


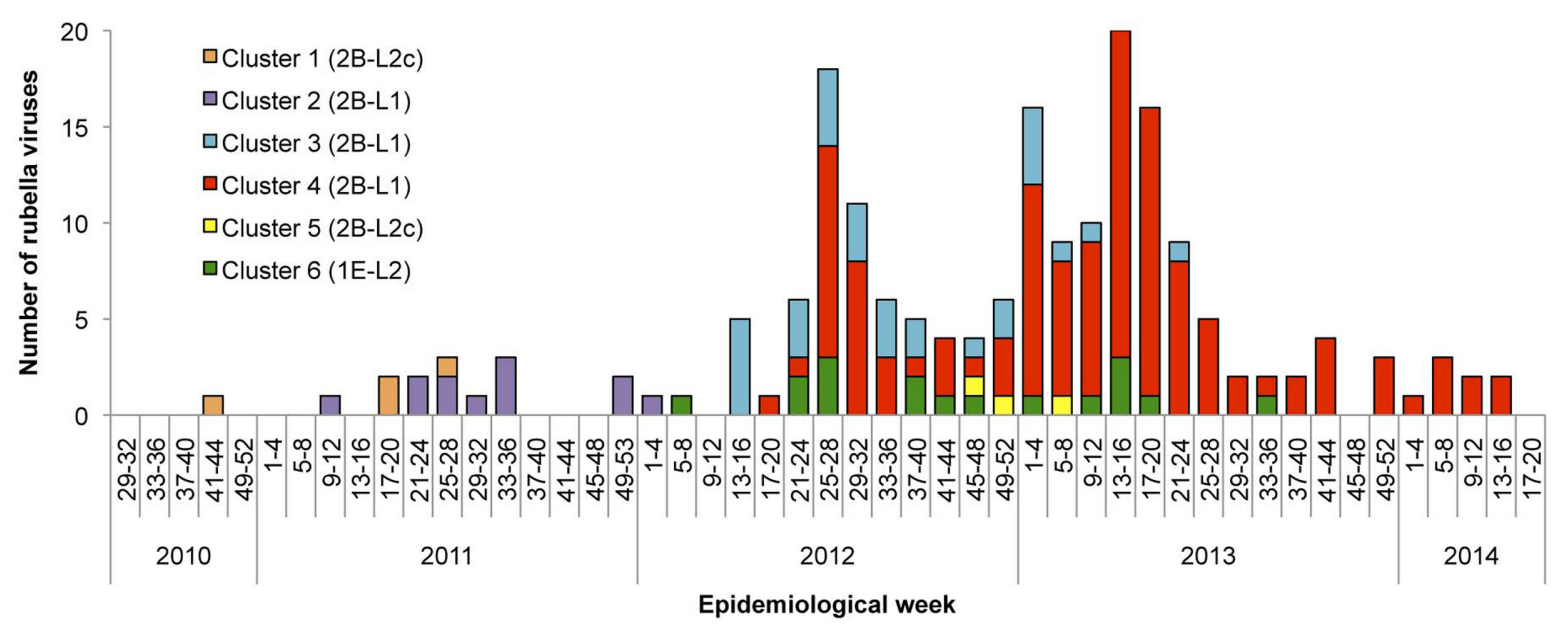

FIGURE 3 | Time course of detection of RV strains by genetic group between 2010 and 2014 in Japan. The number of RV strains according to the genetic clusters defined in Figures 1, 2 are indicated by epidemiological weeks at disease onset or sample collection. The RV strains detected from CRS patients are not included due to a lack of information about the dates of maternal infection.

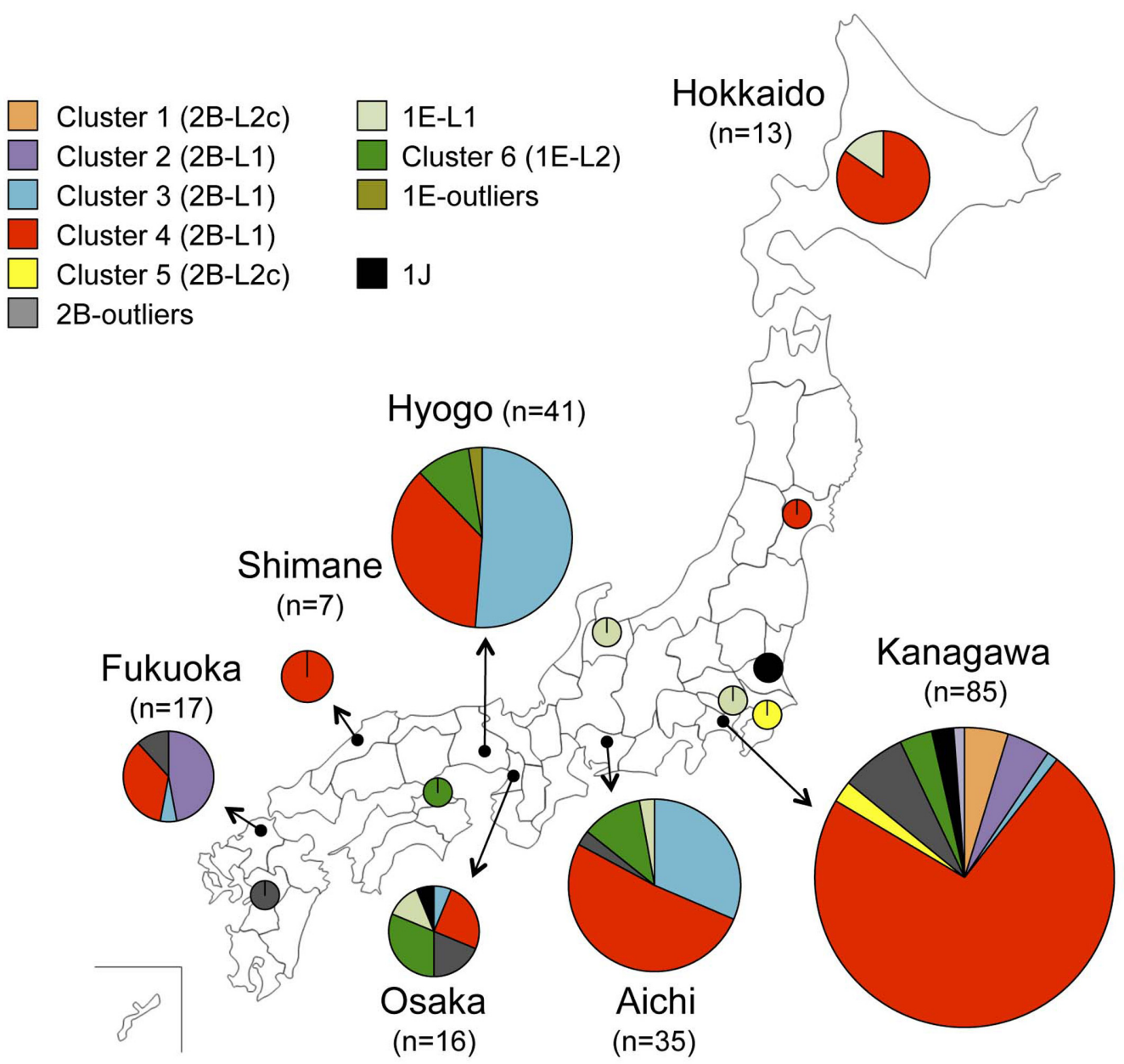

FIGURE 4 | Geographic map showing the location of detection of RV strains by genetic group between 2010 and 2014 in Japan. Detection rates of the genetic clusters of RV defined in Figures 1, 2 by prefecture are indicated by pie charts with the sizes relating to the number of strains. 
Almost all of the strains analyzed were classified into one of four different lineages, 1E-L1, 1E-L2, 2B-L1, or 2B-L2c. The majority of strains belonged to lineage $2 \mathrm{~B}-\mathrm{L} 1$, for which member strains were frequently reported in Southeast and East Asian countries (Tran et al., 2012; Cheng et al., 2013; Zhu et al., 2015; Rivailler et al., 2017). Before the epidemic in Japan, 2B-L1 strains were already circulating in these regions between 2006 and 2010 (Tran et al., 2012; Cheng et al., 2013) and caused a huge outbreak in 2010-2011 in Vietnam (Pham et al., 2013). It was also reported that such strains had been introduced into and spread around mainland China prior to 2011 (Zhu et al., 2015). The rapid spread of RV strains in neighboring countries preceded the introduction of this type of RV strain into Japan. In addition, there were reports that RV strains of the lineages 1E-L1 and 1E-L2 were also circulating in East and Southeast Asian countries (Cheng et al., 2013; Zhu et al., 2015; Rivailler et al., 2017). The RV strains detected in Japan between 2010 and 2014 displayed a variety of genetic backgrounds (i.e., three genotypes, four lineages, and several clusters), suggesting that these strains had been introduced from multiple sources, likely from neighboring countries. Some of countries in the WHO Western Pacific and South-East Asian regions had not introduced a rubellacontaining vaccine into the national immunization program by 2014 (Grant et al., 2015).

Routine immunization coverage of the rubella-containing vaccine during the fiscal years 2011-2013 was $\geq 92 \%$ in two cohorts (National Institute of Infectious Diseases, and Tuberculosis, and Infectious Diseases Control Division, Ministry of Health, Labor, and Welfare, Japan, 2015) and population immunity against rubella in Japan was over $90 \%$ among children (boys and girls) and adult females in 2012 (Tuberculosis and Infectious Diseases Control Division, Ministry of Health, Labor, and Welfare, Japan, and Infectious Disease Surveillance Center, National Institute of Infectious Diseases, 2015). However, an immunization gap still remained for adult males, particularly those aged in their $30 \mathrm{~s}$ to $50 \mathrm{~s}$ who had not received rubella vaccination through the routine immunization program (Tanaka-Taya et al., 2013; Saitoh and Okabe, 2014; Ujiie et al., 2014). As a result of this situation, the introduction of a new RV strain might result in sporadic or small outbreaks, however, it is possible that large outbreaks, such as those seen in 2012-2013, could occur when RV spreads among adult males. This is a result of the policies of many countries including Japan to target rubella vaccination to only adolescent girls or women of childbearing age with the aim of preventing CRS in newborns (Reef and Plotkin, 2012). In these countries, it is thought that filling the immunization gap by introducing supplementary immunizations targeting susceptible populations is required for the maintenance of rubella control or elimination. Furthermore, strengthening rubella vaccination and surveillance on a global scale may be important to interrupt global circulation of these viruses.
According to the framework for verifying elimination of measles and rubella by the Strategic Advisory Group of Experts for measles and rubella (WHO, 2013a), endemic RV transmission is defined as the existence of continuous transmission of indigenous or imported RV that persists for $\geq 12$ months in any defined geographic area. In terms of this definition, it is likely that RV strains within the clusters 3, 4, and 6 have become current endemic strains in Japan because all have been detected for $\geq 12$ months. For verification of rubella elimination in Japan in the future, interruptions in transmission of these strains will need to be confirmed by effective surveillance systems.

The limitation of the present study is that the number of strains analyzed represented approximately 1 per 80 rubella or CRS cases and the sampling was biased to some degree by geographic area. However, our findings provide an overview of the genetic epidemiology of the 2012-2013 rubella epidemic in Japan that will be useful when devising domestic and global strategies for rubella elimination.

\section{AUTHOR CONTRIBUTIONS}

YoM, KK, and MT designed the study. YoM, MM, MKi, MaS, MU, MiS, YuM, MI, YY, DK, TM, KA, CT, SZ, MKa, NO, KO, and MSa determined the nucleotide sequences of the RV strains and analyzed the data. YoM and MT wrote the manuscript. All authors reviewed the manuscript.

\section{FUNDING}

This work was partly supported by a Grant-in-Aid from the Japan Agency for Medical Research and Development, AMED.

\section{ACKNOWLEDGMENTS}

We are grateful to Drs. H. Nagano and M. Okano (Hokkaido Institute of Public Health), H. Minagawa (Aichi Prefectural Institute of Public Health), T. Kurata (Osaka Institute of Public Health), T. Kase (Osaka City University), K. Uchino (Sakai City Institute of Public Health), T. Tanaka (Hidaka General Hospital), and M. Wada (Shimane Prefecture) for their contributions to this study. We also thank Ms. M. Nagai for her technical support.

\section{SUPPLEMENTARY MATERIAL}

The Supplementary Material for this article can be found online at: http://journal.frontiersin.org/article/10.3389/fmicb. 2017.01513/full\#supplementary-material 


\section{REFERENCES}

Abernathy, E. S., Hubschen, J. M., Muller, C. P., Jin, L., Brown, D., Komase, K., et al. (2011). Status of global virologic surveillance for rubella viruses. J. Infect. Dis. 204(Suppl. 1), S524-S532. doi: 10.1093/infdis/jir099

Cheng, W. Y., Wang, H. C., Liu, M. T., and Wu, H. S. (2013). Molecular surveillance of rubella viruses in Taiwan from 2005 to 2011. J. Med. Virol. 85, 745-753. doi: 10.1002/jmv.23451

Cong, H., Jiang, Y., and Tien, P. (2011). Identification of the myelin oligodendrocyte glycoprotein as a cellular receptor for rubella virus. J. Virol. 85, 11038-11047. doi: 10.1128/JVI.05398-11

Dube, M., Rey, F. A., and Kielian, M. (2014). Rubella virus: first calcium-requiring viral fusion protein. PLoS Pathog. 10:e1004530. doi: 10.1371/journal.ppat. 1004530

DuBois, R. M., Vaney, M. C., Tortorici, M. A., Kurdi, R. A., Barba-Spaeth, G., Krey, T., et al. (2013). Functional and evolutionary insight from the crystal structure of rubella virus protein E1. Nature 493, 552-556. doi: 10.1038/ nature 11741

Grant, G. B., Reef, S. E., Dabbagh, A., Gacic-Dobo, M., and Strebel, P. M. (2015). Global progress toward rubella and congenital rubella syndrome control and elimination - 2000-2014. MMWR Morb. Mortal. Wkly. Rep. 64, 1052-1055. doi: 10.15585/mmwr.mm6437a5

Hobman, T. (2013). "Rubella virus," in Fields Virology, 6th Edn, eds D. M. Knipe and P. M. Howley (Philadelphia, PA: Lippincott Williams \& Wilkins), 687-711.

Katow, S. (2004a). Molecular epidemiology of rubella virus in Asia: utility for reduction in the burden of diseases due to congenital rubella syndrome. Pediatr. Int. 46, 207-213. doi: 10.1046/j.1442-200x.2004.01866.x

Katow, S. (2004b). Surveillance of congenital rubella syndrome in Japan, 1978-2002: effect of revision of the immunization law. Vaccine 22, 4084-4091. doi: 10.1016/j.vaccine.2004.03.055

Ministry of Health, Labor, and Welfare, Japan (2017). Number of Vaccinees through the Routine Immunization Program (in Japanese). Available at: http://www mhlw.go.jp/topics/bcg/other/5.html

Mulders, M. N., Rota, P. A., Icenogle, J. P., Brown, K. E., Takeda, M., Rey, G. J., et al. (2016). Global measles and rubella laboratory network support for elimination goals, 2010-2015. Wkly. Epidemiol. Rec. 91, 240-246. doi: 10.15585/mmwr. mm6517a3

National Institute of Infectious Diseases, and Tuberculosis, and Infectious Diseases Control Division, Ministry of Health, Labor, and Welfare, Japan (2015). Rubella and congenital rubella syndrome in Japan, as of June 2015. Infect. Agents Surveill. Rep. 36, 117-118. doi: 10.7883/yoken.JJID.2014.195

National Institute of Infectious Diseases, and Tuberculosis, and Infectious Diseases Control Division, Ministry of Health, Labor, and Welfare, Japan (2016). Measles and rubella/congenital rubella syndrome in Japan, as of March 2016. Infect. Agents Surveill. Rep. 37, 59-60.

Otsuki, N., Abo, H., Kubota, T., Mori, Y., Umino, Y., Okamoto, K., et al. (2011). Elucidation of the full genetic information of Japanese rubella vaccines and the genetic changes associated with in vitro and in vivo vaccine virus phenotypes. Vaccine 29, 1863-1873. doi: 10.1016/j.vaccine.2011.01.016

Pham, V. H., Nguyen, T. V., Nguyen, T. T., Dang, L. D., Hoang, N. H., Nguyen, T. V., et al. (2013). Rubella epidemic in Vietnam: characteristic of rubella virus genes from pregnant women and their fetuses/newborns with congenital rubella syndrome. J. Clin. Virol. 57, 152-156. doi: 10.1016/j.jcv.2013.02.008

Reef, S., and Plotkin, S. (2012). "Rubella vaccine," in Vaccine, 6th Edn, eds S. A. Plotkin, W. Orenstein, and P. Offit (London: Saunders), 688-717.
Rivailler, P., Abernathy, E., and Icenogle, J. (2017). Genetic diversity of currently circulating rubella viruses: a need to define more precise viral groups. J. Gen. Virol. 98, 396-404. doi: 10.1099/jgv.0.000680

Saitoh, A., and Okabe, N. (2014). Recent progress and concerns regarding the Japanese immunization program: addressing the "vaccine gap". Vaccine 32, 4253-4258. doi: 10.1016/j.vaccine.2014.06.022

Tamura, K., and Nei, M. (1993). Estimation of the number of nucleotide substitutions in the control region of mitochondrial DNA in humans and chimpanzees. Mol. Biol. Evol. 10, 512-526.

Tanaka-Taya, K., Satoh, H., Arai, S., Yamagishi, T., Yahata, Y., Nakashima, K., et al. (2013). Nationwide rubella epidemic-Japan, 2013. Morb. Mortal. Wkly. Rep. 62, 457-462.

The Decade of Vaccine Collaboration (2012). Global Vaccine Action Plan 2011-2020. [Webpage on the Internet]. Available at: http://www.who.int/ immunization/global_vaccine_action_plan/GVAP_doc_2011_2020/en/

Tran, D. N., Pham, N. T., Tran, T. T., Khamrin, P., Thongprachum, A., Komase, K., et al. (2012). Phylogenetic analysis of rubella viruses in Vietnam during 2009-2010. J. Med. Virol. 84, 705-710. doi: 10.1002/jmv.23199

Tuberculosis and Infectious Diseases Control Division, Ministry of Health, Labor, and Welfare, Japan, and Infectious Disease Surveillance Center, National Institute of Infectious Diseases (2015). "Chapter 5-Rubella," in Proceedings of the Annual Report 2012 National Epidemiological Surveillance of VaccinePreventable Diseases, Tokyo, 148-153.

Ueda, K., Miyazaki, C., Hidaka, Y., Okada, K., Kusuhara, K., and Kadoya, R. (1995). Aseptic meningitis caused by measles-mumps-rubella vaccine in Japan. Lancet 346, 701-702. doi: 10.1016/S0140-6736(95)92311-X

Ujiie, M., Nabae, K., and Shobayashi, T. (2014). Rubella outbreak in Japan. Lancet 383, 1460-1461. doi: 10.1016/S0140-6736(14)60712-1

WHO (2013a). Framework for verifying elimination of measles and rubella. Wkly. Epidemiol. Rec. 88, 89-99.

WHO (2013b). Rubella virus nomenclature update: 2013. Wkly. Epidemiol. Rec. 88, 337-343.

Wolinsky, J. S., McCarthy, M., Allen-Cannady, O., Moore, W. T., Jin, R., Cao, S. N., et al. (1991). Monoclonal antibody-defined epitope map of expressed rubella virus protein domains. J. Virol. 65, 3986-3994.

Zhu, Z., Cui, A., Wang, H., Zhang, Y., Liu, C., Wang, C., et al. (2012). Emergence and continuous evolution of genotype $1 \mathrm{E}$ rubella viruses in China. J. Clin Microbiol. 50, 353-363. doi: 10.1128/JCM.01264-11

Zhu, Z., Rivailler, P., Abernathy, E., Cui, A., Zhang, Y., Mao, N., et al. (2015). Evolutionary analysis of rubella viruses in mainland China during 2010-2012: endemic circulation of genotype $1 \mathrm{E}$ and introductions of genotype 2B. Sci. Rep. 5:7999. doi: 10.1038/srep07999

Conflict of Interest Statement: The authors declare that the research was conducted in the absence of any commercial or financial relationships that could be construed as a potential conflict of interest.

Copyright (c) 2017 Mori, Miyoshi, Kikuchi, Sekine, Umezawa, Saikusa, Matsushima, Itamochi, Yasui, Kanbayashi, Miyoshi, Akiyoshi, Tatsumi, Zaitsu, Kadoguchi, Otsuki, Okamoto, Sakata, Komase and Takeda. This is an open-access article distributed under the terms of the Creative Commons Attribution License (CC BY). The use, distribution or reproduction in other forums is permitted, provided the original author(s) or licensor are credited and that the original publication in this journal is cited, in accordance with accepted academic practice. No use, distribution or reproduction is permitted which does not comply with these terms. 